Acta Crystallographica Section E

Structure Reports

Online

ISSN 1600-5368

\section{2-Chloro- $N^{\prime}-[(E)-(2-m e t h o x y-1-n a p h t h y l)-$ methylene]benzohydrazide}

\section{Chunbao Tang}

Department of Chemistry, Jiaying University, Meizhou 514015, People's Republic of China

Correspondence e-mail: chunbao_tang@126.com

Received 26 April 2009; accepted 5 May 2009

Key indicators: single-crystal X-ray study; $T=298 \mathrm{~K}$; mean $\sigma(\mathrm{C}-\mathrm{C})=0.006 \AA$;

$R$ factor $=0.062 ; w R$ factor $=0.142 ;$ data-to-parameter ratio $=15.7$.

In the molecule of the title Schiff base compound, $\mathrm{C}_{19} \mathrm{H}_{15} \mathrm{ClN}_{2} \mathrm{O}_{2}$, the dihedral angle between the benzene ring and naphthyl ring system is $77.1(2)^{\circ}$. In the crystal structure, centrosymmetrically related molecules are linked into dimers through pairs of intermolecular $\mathrm{N}-\mathrm{H}$... O hydrogen bonds, generating rings of graph set $R_{2}^{2}(8)$.

\section{Related literature}

For related structures, see: Tang (2007, 2008). For bond-length data, see: Allen et al. (1987). For graph-set analysis, see: Etter et al. (1990); Bernstein et al. (1995).<smiles>COc1ccc2ccccc2c1/C=N/NC(=O)c1ccccc1Cl</smiles>

\section{Experimental}

Crystal data

$\mathrm{C}_{19} \mathrm{H}_{15} \mathrm{ClN}_{2} \mathrm{O}_{2}$

$M_{r}=338.78$
Monoclinic, $P 2_{1} / c$

$a=10.751(2) \AA$

$b=11.405$ (2) $\AA$

$c=14.376(3) \AA$

$\beta=107.794$ (10)

$V=1678.4(6) \AA^{3}$

Data collection

Bruker SMART CCD area-detector diffractometer

Absorption correction: multi-scan (SADABS; Sheldrick, 1996)

$T_{\min }=0.931, T_{\max }=0.938$

Refinement

$R\left[F^{2}>2 \sigma\left(F^{2}\right)\right]=0.062$

$w R\left(F^{2}\right)=0.142$

$S=0.88$

3473 reflections

221 parameters

1 restraint

$Z=4$

Mo $K \alpha$ radiation

$\mu=0.24 \mathrm{~mm}^{-1}$

$T=298 \mathrm{~K}$

$0.30 \times 0.28 \times 0.27 \mathrm{~mm}$

Table 1

Hydrogen-bond geometry $\left(\AA{ }^{\circ}\right)$.

\begin{tabular}{lllll}
\hline$D-\mathrm{H} \cdots A$ & $D-\mathrm{H}$ & $\mathrm{H} \cdots A$ & $D \cdots A$ & $D-\mathrm{H} \cdots A$ \\
\hline $\mathrm{N} 2-\mathrm{H} 2 \cdots \mathrm{O} 2{ }^{\mathrm{i}}$ & $0.90(3)$ & $1.99(3)$ & $2.886(4)$ & $172(4)$ \\
\hline
\end{tabular}

Symmetry code: (i) $-x+1,-y+1,-z$.

Data collection: SMART (Bruker, 2002); cell refinement: SAINT (Bruker, 2002); data reduction: $S A I N T$; $\operatorname{program}(\mathrm{s})$ used to solve structure: SHELXS97 (Sheldrick, 2008); program(s) used to refine structure: SHELXL97 (Sheldrick, 2008); molecular graphics: SHELXTL (Sheldrick, 2008); software used to prepare material for publication: SHELXL97.

Financial support from Jiaying University Research Fund is gratefully acknowledged.

Supplementary data and figures for this paper are available from the IUCr electronic archives (Reference: RZ2320).

\section{References}

Allen, F. H., Kennard, O., Watson, D. G., Brammer, L., Orpen, A. G. \& Taylor, R. (1987). J. Chem. Soc. Perkin Trans. 2, pp. S1-19.

Bernstein, J., Davis, R. E., Shimoni, L. \& Chang, N.-L. (1995). Angew. Chem. Int. Ed. Engl. 34, 1555-1573.

Bruker (2002). SAINT and SMART. Bruker AXS Inc., Madison, Wisconsin, USA.

Etter, M. C., MacDonald, J. C. \& Bernstein, J. (1990). Acta Cryst. B46, 256-262. Sheldrick, G. M. (1996). SADABS. University of Göttingen, Germany.

Sheldrick, G. M. (2008). Acta Cryst. A64, 112-122.

Tang, C.-B. (2007). Acta Cryst. E63, o4841.

Tang, C.-B. (2008). Acta Cryst. E64, o1381. 


\section{supporting information}

Acta Cryst. (2009). E65, o1254 [doi:10.1107/S1600536809016936]

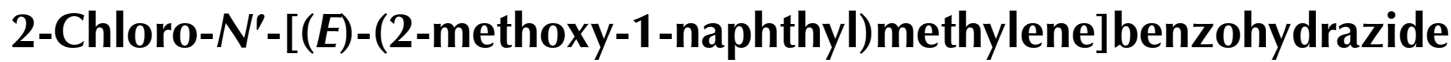

\section{Chunbao Tang}

\section{S1. Comment}

Recently, the author has reported the structures of a few Schiff base compounds (Tang, 2007; Tang 2008). In a continuation of work in this area, the crystal structure of the title compound is reported herein.

In the title compound (Fig. 1), the dihedral angle between the benzene ring and the naphthyl ring system is 77.1 (2) ${ }^{\circ}$. The molecule adopts an $\mathrm{E}$ configuration about the $\mathrm{C}=\mathrm{N}$ bond. All the bond lengths are within normal values (Allen et al., 1987). In the crystal structure (Fig. 2), centrosymmetrically related molecules are linked into dimers through intermolecular $\mathrm{N}-\mathrm{H} \cdots \mathrm{O}$ hydrogen bonds (Table 1), forming rings of graph set $R^{2}{ }_{2}(8)$ (Etter et al., 1990; Bernstein et al., 1995).

\section{S2. Experimental}

2-Methoxy-1-naphthylaldehyde $(0.1 \mathrm{mmol}, 18.6 \mathrm{mg})$ and 2-chlorobenzohydrazide $(0.1 \mathrm{mmol}, 12.6 \mathrm{mg})$ were dissolved in a methanol solution $(20 \mathrm{ml})$. The mixture was stirred at reflux for $10 \mathrm{~min}$ to give a clear colourless solution. Colourless block-like crystals of the compound were formed by slow evaporation of the solvent over several days.

\section{S3. Refinement}

Atom $\mathrm{H} 2$ was located from a difference Fourier map and refined isotropically, with $U_{\text {iso }}$ restrained to $0.08 \AA^{2}$. Other $\mathrm{H}$ atoms were constrained to ideal geometries, with $\mathrm{C}-\mathrm{H}=0.93-0.96 \AA$, and with $U_{\mathrm{iso}}(\mathrm{H})=1.2 U_{\mathrm{eq}}(\mathrm{C})$ or $1.5 U_{\mathrm{eq}}(\mathrm{C})$ for methyl $\mathrm{H}$ atoms. 


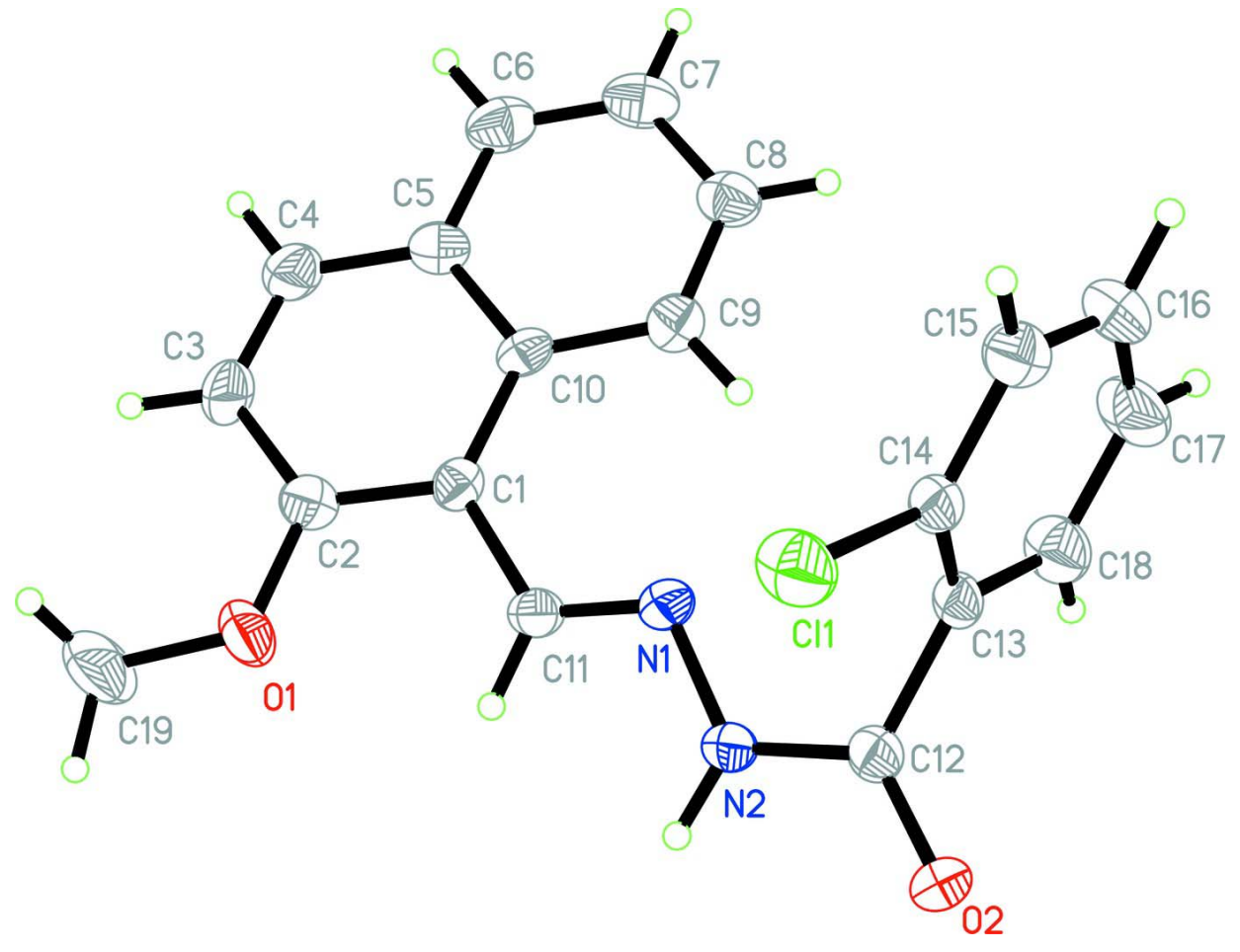

\section{Figure 1}

The molecular structure of the title compound, showing the atom-numbering scheme. Displacement ellipsoids are drawn at the $30 \%$ probability level. 


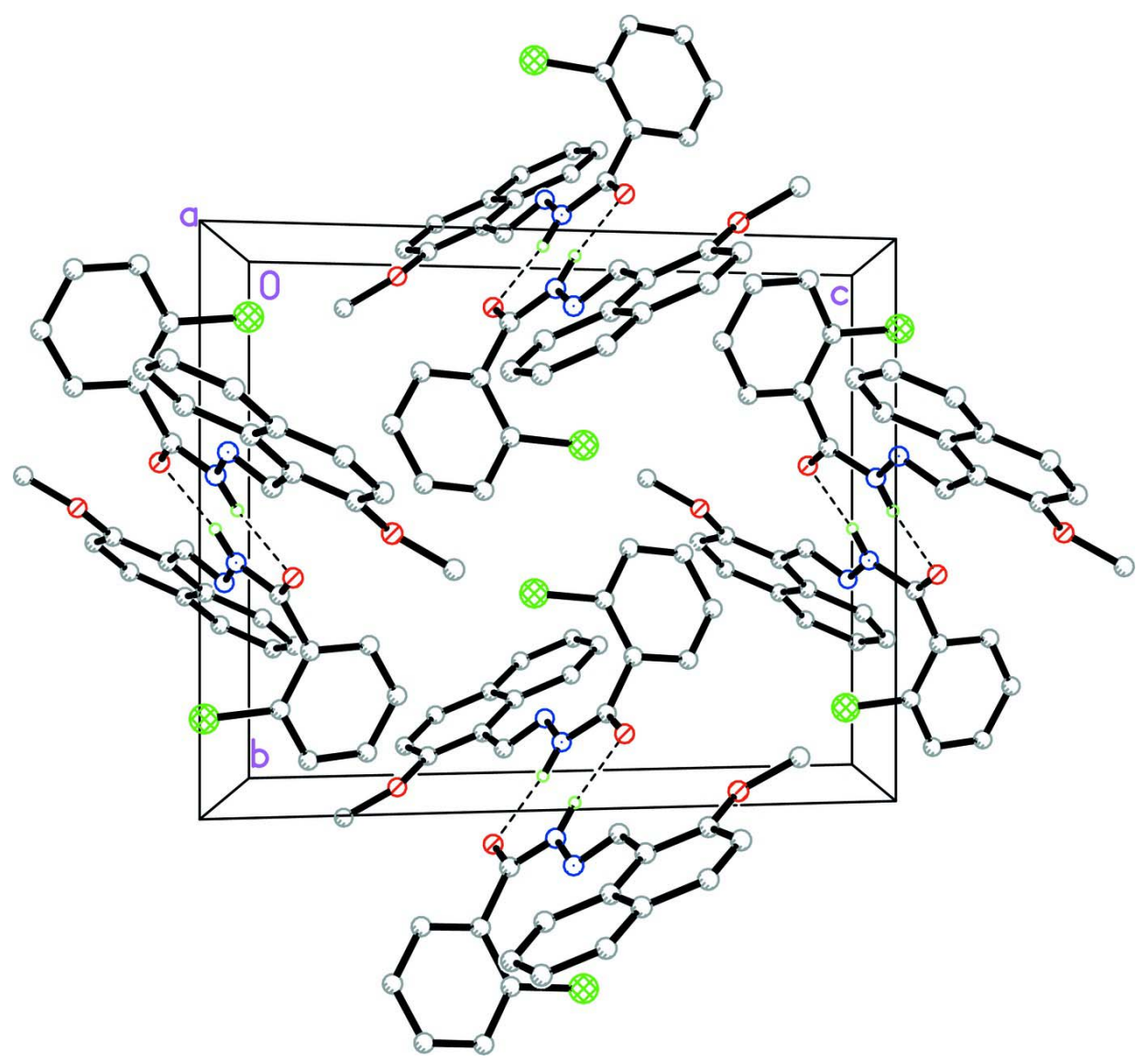

Figure 2

Packing diagram of the title compound viewed along the $a$ axis. Intermolecular hydrogen bonds are drawn as dashed lines. Hydrogen atoms not involved in hydrogen bonding are omitted for clarity.

\section{2-chloro-N'-[(E)-(2-methoxy-1-naphthyl)methylene]benzohydrazide}

Crystal data

$\mathrm{C}_{19} \mathrm{H}_{15} \mathrm{ClN}_{2} \mathrm{O}_{2}$

$M_{r}=338.78$

Monoclinic, $P 2_{1} / c$

Hall symbol: -P 2ybc

$a=10.751(2) \AA$

$b=11.405(2) \AA$

$c=14.376(3) \AA$

$\beta=107.794(10)^{\circ}$

$V=1678.4(6) \AA^{3}$

$Z=4$

\section{Data collection}

Bruker SMART CCD area-detector diffractometer

Radiation source: fine-focus sealed tube Graphite monochromator $\omega$ scans
$F(000)=704$

$D_{\mathrm{x}}=1.341 \mathrm{Mg} \mathrm{m}^{-3}$

Mo $K \alpha$ radiation, $\lambda=0.71073 \AA$

Cell parameters from 394 reflections

$\theta=2.3-24.5^{\circ}$

$\mu=0.24 \mathrm{~mm}^{-1}$

$T=298 \mathrm{~K}$

Block, colourless

$0.30 \times 0.28 \times 0.27 \mathrm{~mm}$

Absorption correction: multi-scan

(SADABS; Sheldrick, 1996)

$T_{\min }=0.931, T_{\max }=0.938$

13186 measured reflections

3473 independent reflections

1295 reflections with $I>2 \sigma(I)$ 


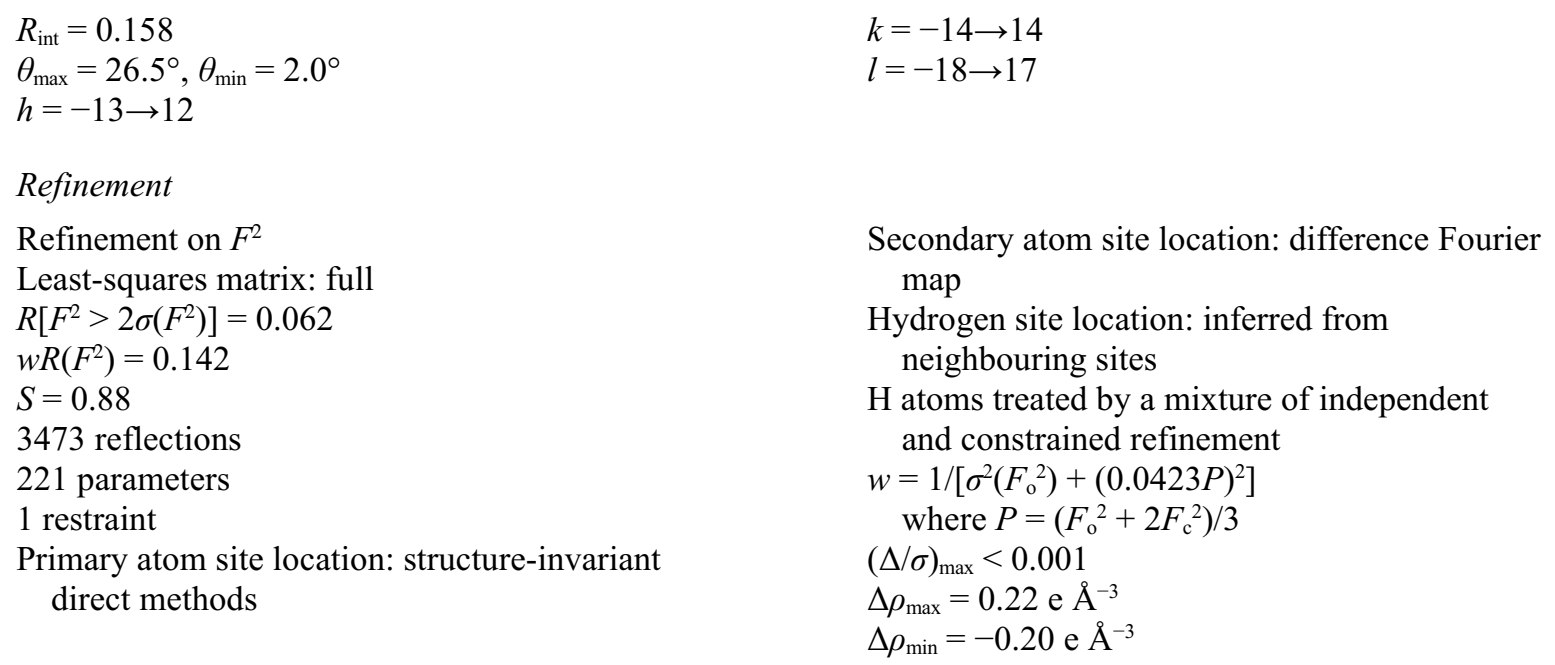

Special details

Geometry. All esds (except the esd in the dihedral angle between two 1.s. planes) are estimated using the full covariance matrix. The cell esds are taken into account individually in the estimation of esds in distances, angles and torsion angles; correlations between esds in cell parameters are only used when they are defined by crystal symmetry. An approximate (isotropic) treatment of cell esds is used for estimating esds involving 1.s. planes.

Refinement. Refinement of $\mathrm{F}^{2}$ against ALL reflections. The weighted $\mathrm{R}$-factor $\mathrm{wR}$ and goodness of fit $\mathrm{S}$ are based on $\mathrm{F}^{2}$, conventional R-factors $\mathrm{R}$ are based on $\mathrm{F}$, with $\mathrm{F}$ set to zero for negative $\mathrm{F}^{2}$. The threshold expression of $\mathrm{F}^{2}>2$ sigma $\left(\mathrm{F}^{2}\right)$ is used only for calculating R-factors ( $\mathrm{gt}$ ) etc. and is not relevant to the choice of reflections for refinement. R-factors based on $\mathrm{F}^{2}$ are statistically about twice as large as those based on F, and R- factors based on ALL data will be even larger.

Fractional atomic coordinates and isotropic or equivalent isotropic displacement parameters $\left(\AA^{2}\right)$

\begin{tabular}{|c|c|c|c|c|}
\hline & $x$ & $y$ & $z$ & $U_{\text {iso }} * / U_{\text {eq }}$ \\
\hline $\mathrm{Cl1}$ & $0.58262(12)$ & $0.13770(9)$ & $0.03814(8)$ & $0.0647(4)$ \\
\hline $\mathrm{N} 2$ & $0.6442(3)$ & $0.4212(3)$ & $-0.0046(2)$ & $0.0427(8)$ \\
\hline O1 & $0.9807(3)$ & 0.5231 & $0.2634(2)$ & $0.0675(9)$ \\
\hline N1 & 0.7735 & 0.3824 & $0.0222(2)$ & $0.0435(9)$ \\
\hline $\mathrm{C} 1$ & $0.9913(4)$ & 0.4199 & 0.1258 & $0.0386(10)$ \\
\hline $\mathrm{O} 2$ & $0.4392(3)$ & $0.3960(2)$ & $-0.10180(18)$ & $0.0516(8)$ \\
\hline $\mathrm{C} 10$ & $1.0644(4)$ & $0.3584(3)$ & $0.0738(3)$ & $0.0397(10)$ \\
\hline $\mathrm{C} 12$ & $0.5539(4)$ & 0.3633 & $-0.0752(3)$ & $0.0416(10)$ \\
\hline C13 & $0.5987(3)$ & 0.2590 & -0.1185 & $0.0379(10)$ \\
\hline $\mathrm{C} 2$ & $1.0565(4)$ & $0.4677(3)$ & $0.2165(3)$ & $0.0474(11)$ \\
\hline $\mathrm{C} 11$ & $0.8515(4)$ & $0.4435(3)$ & $0.0891(3)$ & $0.0413(10)$ \\
\hline H11 & 0.8179 & 0.5057 & 0.1158 & $0.050^{*}$ \\
\hline $\mathrm{C} 5$ & $1.2017(4)$ & 0.3485 & $0.1163(3)$ & 0.0493 (11) \\
\hline $\mathrm{C} 14$ & $0.6132(3)$ & $0.1509(4)$ & $-0.0725(3)$ & $0.0417(10)$ \\
\hline C6 & $1.2771(4)$ & $0.2898(4)$ & $0.0656(4)$ & 0.0627 (13) \\
\hline H6 & 1.3670 & 0.2834 & 0.0940 & $0.075 *$ \\
\hline $\mathrm{C} 9$ & $1.0092(4)$ & 0.3089 & -0.0203 & $0.0480(11)$ \\
\hline H9 & 0.9197 & 0.3150 & -0.0509 & $0.058 *$ \\
\hline $\mathrm{C} 3$ & $1.1932(4)$ & $0.4552(4)$ & $0.2586(3)$ & $0.0581(12)$ \\
\hline $\mathrm{H} 3$ & 1.2352 & 0.4869 & 0.3198 & $0.070 *$ \\
\hline $\mathrm{C} 8$ & $1.0851(4)$ & 0.2527 & $-0.0668(3)$ & $0.0547(12)$ \\
\hline
\end{tabular}




\begin{tabular}{lllll} 
H8 & 1.0462 & 0.2203 & -0.1282 & $0.066^{*}$ \\
C15 & $0.6539(4)$ & $0.0537(4)$ & $-0.1128(3)$ & $0.0568(12)$ \\
H15 & 0.6640 & -0.0183 & -0.0810 & $0.068^{*}$ \\
C7 & $1.2201(5)$ & $0.2428(4)$ & $-0.0240(4)$ & $0.0675(14)$ \\
H7 & 1.2705 & 0.2042 & -0.0567 & $0.081^{*}$ \\
C18 & $0.6229(4)$ & $0.2670(4)$ & $-0.2066(3)$ & $0.0590(13)$ \\
H18 & 0.6119 & 0.3385 & -0.2392 & $0.071^{*}$ \\
C4 & $1.2617(4)$ & $0.3971(4)$ & $0.2094(3)$ & $0.0604(13)$ \\
H4 & 1.3514 & 0.3886 & 0.2375 & $0.072^{*}$ \\
C16 & $0.6793(4)$ & $0.0650(4)$ & $-0.2006(3)$ & $0.0675(14)$ \\
H16 & 0.7074 & 0.0004 & -0.2281 & $0.081^{*}$ \\
C17 & $0.6633(5)$ & $0.1709(5)$ & $-0.2476(3)$ & $0.0726(15)$ \\
H17 & 0.6798 & 0.1779 & -0.3072 & $0.087^{*}$ \\
C19 & $1.0386(5)$ & $0.5842(4)$ & $0.3518(4)$ & $0.1002(19)$ \\
H19A & 1.0830 & 0.5296 & 0.4017 & $0.150^{*}$ \\
H19B & 0.9720 & 0.6240 & 0.3714 & $0.150^{*}$ \\
H19C & 1.1001 & 0.6404 & 0.3423 & $0.150^{*}$ \\
H2 & $0.620(4)$ & $0.483(2)$ & $0.025(3)$ & $0.080^{*}$ \\
\hline
\end{tabular}

Atomic displacement parameters $\left(\AA^{2}\right)$

\begin{tabular}{|c|c|c|c|c|c|c|}
\hline & $U^{11}$ & $U^{22}$ & $U^{33}$ & $U^{12}$ & $U^{13}$ & $U^{23}$ \\
\hline $\mathrm{Cl1}$ & $0.0873(9)$ & $0.0637(8)$ & $0.0513(7)$ & $0.0114(7)$ & $0.0332(6)$ & $0.0104(6)$ \\
\hline N2 & $0.035(2)$ & $0.045(2)$ & $0.047(2)$ & 0.0046 (19) & 0.0109 (17) & $-0.0093(18)$ \\
\hline $\mathrm{O} 1$ & $0.061(2)$ & $0.085(2)$ & 0.0540 (19) & $0.0004(18)$ & 0.0137 (17) & $-0.0316(18)$ \\
\hline N1 & $0.032(2)$ & $0.048(2)$ & $0.048(2)$ & $0.0042(17)$ & 0.0085 (18) & $-0.0042(18)$ \\
\hline $\mathrm{C} 1$ & $0.036(3)$ & $0.035(2)$ & $0.041(3)$ & $-0.003(2)$ & $0.007(2)$ & $0.001(2)$ \\
\hline $\mathrm{O} 2$ & $0.0356(18)$ & 0.0600 (19) & $0.0539(18)$ & $0.0118(15)$ & $0.0059(15)$ & $-0.0056(15)$ \\
\hline $\mathrm{C} 10$ & $0.030(2)$ & $0.045(3)$ & $0.045(3)$ & $0.005(2)$ & $0.012(2)$ & 0.009 (2) \\
\hline $\mathrm{C} 12$ & $0.041(3)$ & $0.048(3)$ & $0.037(2)$ & $0.001(2)$ & $0.015(2)$ & $-0.003(2)$ \\
\hline $\mathrm{C} 13$ & $0.036(3)$ & $0.052(3)$ & $0.028(2)$ & $0.000(2)$ & $0.013(2)$ & $-0.003(2)$ \\
\hline $\mathrm{C} 2$ & $0.046(3)$ & $0.045(3)$ & $0.053(3)$ & $0.000(2)$ & $0.018(2)$ & $-0.003(2)$ \\
\hline $\mathrm{C} 11$ & $0.040(3)$ & $0.040(3)$ & $0.044(3)$ & $0.006(2)$ & $0.014(2)$ & $0.001(2)$ \\
\hline $\mathrm{C} 5$ & 0.049 (3) & $0.043(3)$ & $0.058(3)$ & $0.003(2)$ & $0.020(3)$ & $0.003(2)$ \\
\hline $\mathrm{C} 14$ & $0.039(3)$ & $0.052(3)$ & $0.034(2)$ & $0.003(2)$ & $0.012(2)$ & $-0.004(2)$ \\
\hline C6 & $0.044(3)$ & $0.058(3)$ & $0.084(4)$ & $0.003(3)$ & $0.017(3)$ & $0.003(3)$ \\
\hline C9 & $0.044(3)$ & $0.052(3)$ & $0.048(3)$ & $-0.002(2)$ & $0.015(2)$ & $0.003(2)$ \\
\hline $\mathrm{C} 3$ & $0.045(3)$ & $0.064(3)$ & $0.052(3)$ & $-0.007(2)$ & $-0.004(2)$ & -0.011 \\
\hline $\mathrm{C} 8$ & $0.064(4)$ & $0.051(3)$ & $0.055(3)$ & $0.008(2)$ & $0.027(3)$ & $0.004(2)$ \\
\hline $\mathrm{C} 15$ & $0.063(3)$ & $0.053(3)$ & $0.053(3)$ & 0.007 (2) & $0.016(2)$ & -0.001 \\
\hline $\mathrm{C} 7$ & 0.059 (4) & $0.064(3)$ & $0.091(4)$ & $0.010(3)$ & $0.040(3)$ & $0.000(3)$ \\
\hline $\mathrm{C} 18$ & $0.077(4)$ & $0.058(3)$ & $0.046(3)$ & $0.005(3)$ & $0.024(3)$ & $0.005(2)$ \\
\hline $\mathrm{C} 4$ & $0.045(3)$ & $0.061(3)$ & $0.066(3)$ & $0.004(2)$ & $0.003(3)$ & -0.009 (3) \\
\hline C16 & $0.073(3)$ & 0.069 (4) & $0.062(3)$ & $0.012(3)$ & $0.022(3)$ & $-0.022(3)$ \\
\hline $\mathrm{C} 17$ & $0.097(4)$ & $0.081(4)$ & $0.051(3)$ & $0.015(3)$ & $0.038(3)$ & -0.009 (3) \\
\hline C19 & $0.091(4)$ & $0.105(4)$ & $0.097(4)$ & $0.004(3)$ & $0.018(3)$ & $-0.064(4)$ \\
\hline
\end{tabular}


Geometric parameters $\left(\AA,{ }^{\circ}\right)$

\begin{tabular}{|c|c|c|c|}
\hline $\mathrm{C} 11-\mathrm{C} 14$ & $1.726(4)$ & $\mathrm{C} 6-\mathrm{C} 7$ & $1.356(6)$ \\
\hline $\mathrm{N} 2-\mathrm{C} 12$ & $1.344(5)$ & C6- 6 6 & 0.9300 \\
\hline $\mathrm{N} 2-\mathrm{N} 1$ & $1.395(4)$ & $\mathrm{C} 9-\mathrm{C} 8$ & $1.362(5)$ \\
\hline $\mathrm{N} 2-\mathrm{H} 2$ & $0.90(3)$ & $\mathrm{C} 9-\mathrm{H} 9$ & 0.9300 \\
\hline $\mathrm{O} 1-\mathrm{C} 2$ & $1.361(4)$ & $\mathrm{C} 3-\mathrm{C} 4$ & $1.342(5)$ \\
\hline $\mathrm{O} 1-\mathrm{C} 19$ & $1.416(4)$ & $\mathrm{C} 3-\mathrm{H} 3$ & 0.9300 \\
\hline $\mathrm{N} 1-\mathrm{C} 11$ & $1.273(4)$ & $\mathrm{C} 8-\mathrm{C} 7$ & $1.398(6)$ \\
\hline $\mathrm{C} 1-\mathrm{C} 2$ & $1.390(5)$ & $\mathrm{C} 8-\mathrm{H} 8$ & 0.9300 \\
\hline $\mathrm{C} 1-\mathrm{C} 10$ & $1.424(5)$ & $\mathrm{C} 15-\mathrm{C} 16$ & $1.377(5)$ \\
\hline $\mathrm{C} 1-\mathrm{C} 11$ & $1.458(5)$ & C15-H15 & 0.9300 \\
\hline $\mathrm{O} 2-\mathrm{C} 12$ & $1.232(4)$ & $\mathrm{C} 7-\mathrm{H} 7$ & 0.9300 \\
\hline $\mathrm{C} 10-\mathrm{C} 9$ & $1.418(5)$ & $\mathrm{C} 18-\mathrm{C} 17$ & $1.375(5)$ \\
\hline $\mathrm{C} 10-\mathrm{C} 5$ & $1.419(5)$ & C18-H18 & 0.9300 \\
\hline $\mathrm{C} 12-\mathrm{C} 13$ & $1.490(5)$ & $\mathrm{C} 4-\mathrm{H} 4$ & 0.9300 \\
\hline $\mathrm{C} 13-\mathrm{C} 18$ & $1.371(5)$ & $\mathrm{C} 16-\mathrm{C} 17$ & $1.369(6)$ \\
\hline $\mathrm{C} 13-\mathrm{C} 14$ & $1.385(5)$ & $\mathrm{C} 16-\mathrm{H} 16$ & 0.9300 \\
\hline $\mathrm{C} 2-\mathrm{C} 3$ & $1.415(5)$ & C17-H17 & 0.9300 \\
\hline C11-H11 & 0.9300 & C19-H19A & 0.9600 \\
\hline $\mathrm{C} 5-\mathrm{C} 4$ & $1.409(5)$ & C19-H19B & 0.9600 \\
\hline $\mathrm{C} 5-\mathrm{C} 6$ & $1.413(5)$ & $\mathrm{C} 19-\mathrm{H} 19 \mathrm{C}$ & 0.9600 \\
\hline $\mathrm{C} 14-\mathrm{C} 15$ & $1.382(5)$ & & \\
\hline $\mathrm{C} 12-\mathrm{N} 2-\mathrm{N} 1$ & $118.8(3)$ & $\mathrm{C} 8-\mathrm{C} 9-\mathrm{H} 9$ & 119.5 \\
\hline $\mathrm{C} 12-\mathrm{N} 2-\mathrm{H} 2$ & $119(3)$ & $\mathrm{C} 10-\mathrm{C} 9-\mathrm{H} 9$ & 119.5 \\
\hline $\mathrm{N} 1-\mathrm{N} 2-\mathrm{H} 2$ & $122(3)$ & $\mathrm{C} 4-\mathrm{C} 3-\mathrm{C} 2$ & $119.3(4)$ \\
\hline $\mathrm{C} 2-\mathrm{O} 1-\mathrm{C} 19$ & $120.5(3)$ & $\mathrm{C} 4-\mathrm{C} 3-\mathrm{H} 3$ & 120.3 \\
\hline $\mathrm{C} 11-\mathrm{N} 1-\mathrm{N} 2$ & $114.0(3)$ & $\mathrm{C} 2-\mathrm{C} 3-\mathrm{H} 3$ & 120.3 \\
\hline $\mathrm{C} 2-\mathrm{C} 1-\mathrm{C} 10$ & $119.0(4)$ & $\mathrm{C} 9-\mathrm{C} 8-\mathrm{C} 7$ & $121.3(4)$ \\
\hline $\mathrm{C} 2-\mathrm{C} 1-\mathrm{C} 11$ & $115.8(4)$ & $\mathrm{C} 9-\mathrm{C} 8-\mathrm{H} 8$ & 119.4 \\
\hline $\mathrm{C} 10-\mathrm{C} 1-\mathrm{C} 11$ & $125.1(4)$ & $\mathrm{C} 7-\mathrm{C} 8-\mathrm{H} 8$ & 119.4 \\
\hline $\mathrm{C} 9-\mathrm{C} 10-\mathrm{C} 5$ & $117.1(4)$ & $\mathrm{C} 16-\mathrm{C} 15-\mathrm{C} 14$ & $119.1(4)$ \\
\hline $\mathrm{C} 9-\mathrm{C} 10-\mathrm{C} 1$ & $124.0(4)$ & $\mathrm{C} 16-\mathrm{C} 15-\mathrm{H} 15$ & 120.4 \\
\hline $\mathrm{C} 5-\mathrm{C} 10-\mathrm{C} 1$ & $118.8(4)$ & $\mathrm{C} 14-\mathrm{C} 15-\mathrm{H} 15$ & 120.4 \\
\hline $\mathrm{O} 2-\mathrm{C} 12-\mathrm{N} 2$ & $120.6(4)$ & $\mathrm{C} 6-\mathrm{C} 7-\mathrm{C} 8$ & $119.6(4)$ \\
\hline $\mathrm{O} 2-\mathrm{C} 12-\mathrm{C} 13$ & $122.2(4)$ & $\mathrm{C} 6-\mathrm{C} 7-\mathrm{H} 7$ & 120.2 \\
\hline $\mathrm{N} 2-\mathrm{C} 12-\mathrm{C} 13$ & $117.2(4)$ & $\mathrm{C} 8-\mathrm{C} 7-\mathrm{H} 7$ & 120.2 \\
\hline $\mathrm{C} 18-\mathrm{C} 13-\mathrm{C} 14$ & $118.4(4)$ & $\mathrm{C} 13-\mathrm{C} 18-\mathrm{C} 17$ & $121.1(4)$ \\
\hline $\mathrm{C} 18-\mathrm{C} 13-\mathrm{C} 12$ & $120.7(4)$ & $\mathrm{C} 13-\mathrm{C} 18-\mathrm{H} 18$ & 119.5 \\
\hline $\mathrm{C} 14-\mathrm{C} 13-\mathrm{C} 12$ & $120.9(3)$ & $\mathrm{C} 17-\mathrm{C} 18-\mathrm{H} 18$ & 119.5 \\
\hline $\mathrm{O} 1-\mathrm{C} 2-\mathrm{C} 1$ & $116.1(4)$ & $\mathrm{C} 3-\mathrm{C} 4-\mathrm{C} 5$ & $122.0(4)$ \\
\hline $\mathrm{O} 1-\mathrm{C} 2-\mathrm{C} 3$ & $122.4(4)$ & $\mathrm{C} 3-\mathrm{C} 4-\mathrm{H} 4$ & 119.0 \\
\hline $\mathrm{C} 1-\mathrm{C} 2-\mathrm{C} 3$ & $121.4(4)$ & $\mathrm{C} 5-\mathrm{C} 4-\mathrm{H} 4$ & 119.0 \\
\hline $\mathrm{N} 1-\mathrm{C} 11-\mathrm{C} 1$ & $122.7(4)$ & $\mathrm{C} 17-\mathrm{C} 16-\mathrm{C} 15$ & $120.3(4)$ \\
\hline $\mathrm{N} 1-\mathrm{C} 11-\mathrm{H} 11$ & 118.6 & $\mathrm{C} 17-\mathrm{C} 16-\mathrm{H} 16$ & 119.8 \\
\hline $\mathrm{C} 1-\mathrm{C} 11-\mathrm{H} 11$ & 118.6 & $\mathrm{C} 15-\mathrm{C} 16-\mathrm{H} 16$ & 119.8 \\
\hline $\mathrm{C} 4-\mathrm{C} 5-\mathrm{C} 6$ & $120.5(4)$ & $\mathrm{C} 16-\mathrm{C} 17-\mathrm{C} 18$ & $120.0(4)$ \\
\hline
\end{tabular}




$\begin{array}{llll}\mathrm{C} 4-\mathrm{C} 5-\mathrm{C} 10 & 119.4(4) & \mathrm{C} 16-\mathrm{C} 17-\mathrm{H} 17 & 120.0 \\ \mathrm{C} 6-\mathrm{C} 5-\mathrm{C} 10 & 120.1(4) & \mathrm{C} 18-\mathrm{C} 17-\mathrm{H} 17 & 120.0 \\ \mathrm{C} 15-\mathrm{C} 14-\mathrm{C} 13 & 121.1(4) & \mathrm{O} 1-\mathrm{C} 19-\mathrm{H} 19 \mathrm{~A} & 109.5 \\ \mathrm{C} 15-\mathrm{C} 14-\mathrm{C} 11 & 119.3(3) & \mathrm{O} 1-\mathrm{C} 19-\mathrm{H} 19 \mathrm{~B} & 109.5 \\ \mathrm{C} 13-\mathrm{C} 14-\mathrm{C} 11 & 119.5(3) & \mathrm{H} 19 \mathrm{~A}-\mathrm{C} 19-\mathrm{H} 19 \mathrm{~B} & 109.5 \\ \mathrm{C} 7-\mathrm{C} 6-\mathrm{C} 5 & 120.9(4) & \mathrm{O} 1-\mathrm{C} 19-\mathrm{H} 19 \mathrm{C} & 109.5 \\ \mathrm{C} 7-\mathrm{C} 6-\mathrm{H} 6 & 119.6 & \mathrm{H} 19 \mathrm{~A}-\mathrm{C} 19-\mathrm{H} 19 \mathrm{C} & 109.5 \\ \mathrm{C} 5-\mathrm{C} 6-\mathrm{H} 6 & 119.6 & \mathrm{H} 19 \mathrm{~B}-\mathrm{C} 19-\mathrm{H} 19 \mathrm{C} & 109.5 \\ \mathrm{C} 8-\mathrm{C} 9-\mathrm{C} 10 & 121.1(4) & & \end{array}$

Hydrogen-bond geometry $\left(A,{ }^{\circ}\right)$

\begin{tabular}{lllll}
\hline$D-\mathrm{H} \cdots A$ & $D-\mathrm{H}$ & $\mathrm{H} \cdots A$ & $D \cdots A$ & $D-\mathrm{H} \cdots A$ \\
\hline $\mathrm{N} 2-\mathrm{H} 2 \cdots \mathrm{O} 2^{\mathrm{i}}$ & $0.90(3)$ & $1.99(3)$ & $2.886(4)$ & $172(4)$ \\
\hline
\end{tabular}

Symmetry code: (i) $-x+1,-y+1,-z$. 